\title{
The proportion of various graphs in graph-designs
}

\author{
Richard M. Wilson
}

\begin{abstract}
Austratur. Let $\mathcal{G}$ be a family of simple gruphs, $\Lambda \mathcal{G}$-design on $n$ points is a decorrposition of the thges of $K_{n}$ irto copius of graphs in $\mathcal{G}$. In case that $\mathcal{G}$ consists of compltte graphs $K_{k}$ with $k$ in some set $K$ of positive integer's, such a $\mathcal{G}$-dosign is called a pairwise balanced design (FBL) on moints wich block sizes from $K$. Ilere we arc concernted with the possible proportions of the nurmbers of copies of graphs $G \in \mathcal{G}$ that appear in decompositions for large r. We oxtend a resuh of Colbourn and Rödl on PLDs to $\mathcal{G}$-dusigns, and give a further result, on the possible numbers of copits of $G$ in in $G$-destign containing each vertex of the compltite graph $K_{n}$.
\end{abstract}

\section{Introduction}

Por a positive integer $n$ and a sel $K$ of positive integers, a 2-(n, $K, I)$ design consiste of a set $X$ of $n$ points and a farnily $\mathcal{A}$ of subsets of $X$, called blocks, so that $|A| \in K$ for every $A \in \mathcal{A}$, and every subset $\{x, y\}$ of two points in $X$ is contained in a unique momber of $\mathcal{A}$. Theso may also be called pairwise balanced designs ( $\mathrm{PB} \perp \mathrm{s})$ with block sizes in $K$.

We use $\alpha(K)$ for the gcd (greatest common divisor) of $\{k-1: k \in K\}$ and $\beta(K)$ for tho gcd of $\{k(k-1): k \in K\}$. It is known, see $[4 \mid$, that $2-(n, K, 1)$ designs exist for all integers $n$ that are sufficiently large with respect to $K$ and such that

$$
\begin{aligned}
n-1 \equiv 0 & (\bmod \alpha(K)), \\
n(n-1): \equiv 0 & (\bmod \beta(K)) .
\end{aligned}
$$

These congruences are nocessary conditions for the existcnco of a $2-(n, K, 1)$ design for any $n$.

'The Collowing theorem was proved by Colbourn and Rödl in [2].

THEORFM 1.1. Let $K=\left\{k_{1}, k_{2}, \ldots, k_{2}\right\}$ be given, where the integors $k_{i}$ aro distinet and at least 2 . Let $p_{1}, p_{2}, \ldots, p_{2}$ be nonnegative real numbers that squ to 1 , and let $\epsilon>0$. For cvery suffecently larye integer $n$ satisfying $(1,1)$, there exists a $2-(v, K, 1)$ design in which the proportion of blocks of size $k_{i}$ is within $c$ of $p_{i}$, simultaneously for all $i=1,2, \ldots, \ell$.

We give another proof of this theorem. It is no extra work to prove an $\mathrm{txtension}$ of their result to graph-desigris.

1991 Mathematics Subject Chassification. Prinary 05B05, 05C70; Sncondiny 05C35, 05[30.

Key words and phrases, griph designs, complete graph, decomposilionts.

The author was supported in part by NSH Grant \#0555755. 
Lel $\mathcal{G}$ be a family of simple graphs. A $\mathcal{G}$-decomposition of a graph $I I$ is a set $\mathcal{D}$ of edge-disjoint subgraphs of $I I$, each subgraph in $\mathcal{D}$ isornorphic to a member of $\mathcal{G}$, so that every edge belongs to exactly one rncmber of $\mathcal{D}$. A $\mathcal{G}$-design on $n$ points is a $\mathcal{G}$-rlecomposition of the complete graph $K_{n}$.

Wo use $\alpha(\mathcal{G})$ for the ged of the degrees of the verlices of graphs in $\mathcal{G}$, and $\beta(\mathcal{G})$ for the god of $\{2|E(G)|: G \in \mathcal{G}\}$. It is krown $[3]$ that $\mathcal{G}$-designs on $n$ points cxist for all sufficiently large (with respect to $\mathcal{G}$ ) integers $n$ salisfying

$$
\begin{aligned}
n-1 \equiv 0 & (\bmod \alpha(\mathcal{G})), \\
n(n-1) \equiv 0 & (\bmod \beta(\mathcal{G})) .
\end{aligned}
$$

The congruences (1.2) are necossary conditions for the existence of $\mathcal{G}$-designs on $n$ points for any $n$. In general, if there oxists a $\mathcal{G}$-decomposition of $I I$, then

$$
\begin{array}{ll}
\alpha(\{H\}) \equiv 0 & (\bmod \alpha(\mathcal{G})), \\
\beta(\{I I\}) \equiv 0 & (\bmod \beta(\mathcal{G})) .
\end{array}
$$

THrothim 1.2. Let $\mathcal{G}=\left\{G_{1}, G_{2}, \ldots, G_{\ell}\right\}$ be given, where the grophs $G_{1}$ are pairuise nonisomorphic: and where each has at least one edge. Lel $p_{1}, p_{2}, \ldots, p_{\ell}$ be nonnegatise real numbers that sum to 1 , and let $c>0$. For every sufficiently large integer $n$ satisfying the congruences (1.2), there exists a $\mathcal{G}$-design in which the proportion of copies of $G_{i}$ used in the decomposition is within $\epsilon$ of $p_{i}$ for all $i=1,2, \ldots, \ell$.

It should be clear that Theorem 1.1 is the consequence of Theorem 1.2, when we take $G_{i}$ to be a completo graph orl $k_{i}$ points. Theorem 1.2 and a corollary will be proved in Section 2. In Section 3, we prove the following theorem. It is strongcr than Theorem 1.2.

Tulsolkim 1.3. Let $\mathcal{G}=\left\{G_{1}, G_{2}, \ldots, G_{\ell}\right\}$ be given, where the graphs $G_{i}$ are pairmise nonisomorphic and where each has at least one edge. Let $p_{1}, p_{2}, \ldots, p_{\ell}$ be nonnegative ral nambers that sum to 1 , and let $\epsilon>0$. For every sufficiently large integer $n$ salisfing the congruences (1.2), there exists a $\mathcal{G}$-design in which for every point $x$, the proportion of copies of $G_{i}$ that appear in the decomposition and that contain $x$ is within $c$ of $p_{i}$ for all $i=1,2, \ldots, \ell$.

\section{Proof of Theorem 1.2}

Assume the hypothesis and notation of Theorem 1.2. It is sufficient to prove the theorem in the case that the $p_{i}$ 's are rational numbers. Suppose that $p_{i} \cdot s_{i} / t$ (with a common denominator $t$ ) and that $t$ is large enough so that $1 / t<\epsilon$.

Let $I_{0}$ be the vertex-disjoint union of $s_{i}$ copies of $C_{i}, i=1,2 \ldots, \ell$. Let $H_{i}$ bo the vertex-disjoint union of $I_{0}$ and one additional copy of $G_{i}, i \cdot: 1,2, \ldots, \ell$, and let $\mathcal{H}=\left\{H_{0}, H_{1}, \ldots, H_{\ell}\right\}$. In any graph $I_{i}$, the proportion of copies of $G_{i}$ that appear is one of $s_{i} /(t+1), s_{i} / l$, or $\left(s_{i}+1\right) /(t+1)$. Fvery $\mathcal{H}$-design immodiatcly gives us a $\mathcal{G}$-design, in which the proportion of copies of $G_{i}$ that appcar is botwon $s_{i} /(t+1)$ and $\left(s_{i}+1\right) /(t+1)$, and this is within $\epsilon$ of $p_{i}$.

From [3], $\mathcal{H}$-designs on $n$ points exist for all large integers $n$ satisfying

$$
\begin{aligned}
n \ldots 1: \equiv 0 & (\bmod \alpha(\mathcal{H})), \\
n(n-1) \equiv 0 & (\bmod \beta(\mathcal{H})),
\end{aligned}
$$


We claim that

$$
\alpha(\mathcal{H})=\alpha(\mathcal{G}) \quad \text { and } \quad \beta(\mathcal{H}) \cdots \beta(\mathcal{G}),
$$

i.e. that the congruences (1.2) and (2.1) are identical. This will complete the proof of Theorem 1.2.

The set, of degrees of vertices in graphs in $\mathcal{H}$ is identical with the set of degrees of vertices in graphs in $\mathcal{G}$, so the left hand equation in $(2.2)$ is trivial. It is also trivial that $\beta(\mathcal{G})$ divides $\beta(\mathcal{H})$. Finally, since $\beta(\mathcal{H})$ divides $2\left|E\left(H_{j}\right)\right|$ for all $j$, it divides the difference

$$
2\left|E\left(H_{i}\right)\right|-\cdots-2\left|E\left(H_{0}\right)\right|=2\left|E\left(G_{i}\right)\right|
$$

for each $i$. Hence $\beta(\mathcal{H})$ divides the ged of $2\left|E\left(G_{i}\right)\right|, i=1,2 \ldots, \ell$, which is $\beta(\mathcal{G})$. This establishes the right hand equation in (2.2).

By a $G_{\text {mpacking }} \mathcal{P}$ in $K_{n}$ we mean a set of edge-disjoint isomorphic copies of $G$ in $K_{n}$.

Corolitary 1. Let $G$ be a simple graph with at least one edge, and let $\epsilon>0$ be given. For every sufficiently large integer $n$, there exists a $G-$ packing $\mathcal{P}$ of $K_{n}$ so that the ratio of the number edges that occur in copies of $G$ in $\mathcal{P}$ to $n(n-1) / 2$ is more than $1-\epsilon$.

Proof. Apply Theorem 1.2 with $G_{1}=G$, with $G_{2}$ a graph with a single edge, $p_{1}=1$, and $p_{2}=0$. For $\mathcal{G}=\left\{G_{1}, G_{2}\right\}$, we have $\alpha(\mathcal{G})=1$ and $\beta(\mathcal{G})=2$, so all integers $n$ satisfy the congruences (1.2) in this case.

A much stronger result about packings of complete graphs into $K_{\psi_{t}}$ will appear in $[\mathbf{1}]$.

\section{Proof of Theorem 1.3}

$A s$ in Section 2 , we use the fact, that if we have an $\mathcal{A}$ decomposition $\mathcal{D}_{B 3}$ of each graph $B \in \mathcal{B}$, and a $\mathcal{B}$-decomposition of a graph $H$, then we naturally obtain an $\mathcal{A}$-decomposition $\mathcal{D}$ of $H$, namely

$$
\mathcal{D}=\bigcup_{B \in \mathcal{B}} \mathcal{D}_{B B}
$$

Let $A \in A$ and suppose that for every vertex $x$ of a graph $B \in \mathcal{B}$, the ratio of the number of copies of $A$ in $\mathcal{D}_{B}$ that contain $x$ to the total number of graph in $\mathcal{D}_{B 3}$ that contain $x$ is within $\epsilon$ of a number $p$. Then for every vertex $y$ of $H$, the ratio of the number of copies of $A$ in $D$ that contain $y$ to the total number of graph in $\mathcal{D}$ that contain $y$ is within $\epsilon$ of $p$.

It is sufficient to prove 'Theorem 1.3 in the case that the $p_{i}$ 's are rational numbers, and we may also assume that they are positive. Suppose that $p_{i}=s_{i} / t$ (with a common denomination $t$, and where $s_{i}>0$ for all $i$ ) and that $t$ is large enough so that $1 / t<\epsilon$.

Let $u_{i}$ be the number of vertices of $G_{i}$. Let $J$ be the edge disjoint union of $C s_{i} / u_{i}$ coples of $G_{i}, i=1,2, \ldots, \ell$, where $C \geq 2$ is an integer chosen so that $C s_{i} / u_{i}$ is an integer for each $i$.

Label the vertices of $J$ with positive integers in the range from 1 to $N$ for some integer $N$ so that the absolute values of the differences of the labels on adjacent vertices are distinct. For example, an (inefficient) way to do this is to use labels $2^{0}, 2^{1}, 2^{2}, \ldots, 2^{v \cdots 1}$ in any order, where $v$ is the nimber of vertices of $J$; here $N=$ 
$2^{y-1}$. Identify the vertices of $f$ with their labels in the (additive) group $Z_{2 N+1}$ of integers modulo $2 N+1$, so thai $J$ is now a subgraph of the complete graph on veriex set $Z_{2 N+1}$.

Lei $L_{0}$ be the union of all transtations $J+a, a \in Z_{2 N+x}$. The condition on absolute values of the differences of labels ensures that the graphs $J+a$ are pairwise edge disjoint. Because if $x, y \in Z_{2 N+1}$ are adjacent in both $J+a$ and $J+b$, then $x-a, y-a$ are adjacent in $J$ and $x-b, b,-b$ are adjacent in $J$; if these are not the ends of the same edge of $J$, then $d \ldots+(x-y)$ modulo $2 N+1$ appears as the difference of the labels of the ends of two edges of $J$; but then $|d|$ is the difference of the labels of the ends of two edges of $J$.

So $L_{0}$ admits a decomposition into $2 N+1$ copies of $J$, and thern we obtain a $\mathcal{G}$-decomposition $\mathcal{D}_{0}$ of $L_{0}$. The number of translates of a single copy of $G_{i}$ that contain any given point $x \in Z_{2 N+1}$ is $u_{i}$, so the number of copies of $G_{i}$ in $\mathcal{D}_{0}$ that contain any point $x$ is $C s_{i}$. The total number of graphs in $\mathcal{D}_{0}$ that contain $x$ is $C t$.

Let $L_{j}$ be obtained from $L_{0}$ by deleting the edges of one copy of $G_{j}$ from $L_{0}$, $i=1,2, \ldots, \ell$. Of course, $L_{j}$ has a $\mathcal{G}$-decomposition $\mathcal{D}_{j}$ obtained by deleting that one copy of $G_{j}$ from $D$. Wach point $x \in Z_{2 N+1}$ is contained in either $C s_{i}$ or $C s_{i}-1$ copies of $G_{i}$ in $\mathcal{D}_{j}$; the total number of graphs in $\mathcal{D}_{j}$ that contain $x$ is $C t$ or $C t \cdots 1$. In any case, the proportion of copies of $G_{i}$ among the graphs in $\mathcal{D}_{j}$ that contain $x$ is between $\left(C s_{i}-1\right) /(C t)$ and $\left(C s_{i}\right) /(C t-1)$, and is within $1 / t<\epsilon$ of $p_{i}$.

Let $\mathcal{L}=\left\{L_{0}, L_{1}, \ldots, L_{\ell}\right\}$. From [2], $\mathcal{L}$-designs on $n$ poirnts exist for all large integers $n$ satisfying

$$
\begin{aligned}
n-1 \equiv 0 & (\bmod \alpha(\mathcal{L})), \\
n(n-1)=0 & (\bmod \beta(\mathcal{L})), .
\end{aligned}
$$

From a $\mathcal{L}_{m}$-design, we obtain a $\mathcal{G}$-design using the $\mathcal{G}$-decompositions of $L_{i}$ described above. For any point $y$, the proportion of copies of $G_{i}$ among the graphs in the $\mathcal{G}$-decomposition that contain $y$ will be within $t$ of $p_{i}$.

We claim that:

$$
\alpha(\mathcal{L})=\alpha(\mathcal{G}) \quad \text { and } \quad \beta(\mathcal{L})=\beta(\mathcal{G})
$$

i.e. that the congruences (1.3) and (3.1) are identical. This will cornplete the proof of 'Theorem 1.3 .

Firsi, since each $L_{i}$ has a $\mathcal{G}$ decomposition, $\alpha(\mathcal{G})$ divides $\alpha\left(\left\{L_{i}\right\}\right)$ and $\beta(\mathcal{G})$ divides $\beta\left(\left\{L_{i}\right\}\right)$ for each $\dot{b}=1,2, \ldots, \ell$. Ferace $\alpha(\mathcal{G})$ divides $\alpha(\mathcal{L})$ and $\beta(\mathcal{G})$ divides $\beta(\mathcal{L})$.

If there is a vertex of degree $d$ in some $G_{i}$, then, since one copy of $G_{i}$ was deleted

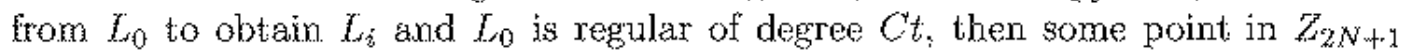
has degree $C_{t}$ in $L_{0}$ and degree $C t \cdots \cdots$ ir $L_{i}$. Then $\alpha(\mathcal{L})$ divides these dagrees and so divides the difference $d$. Since this is true for the degree $d$ of every vertex of any graph in $\mathcal{G}, \alpha(\mathcal{L})$ divides $\alpha(\mathcal{G})$. Also, $\beta(\mathcal{L})$ divides $2\left|E\left(L_{0}\right)\right|$ and $2\left|E\left(L_{i}\right)\right|$, so it divides $2\left|E\left(L_{0}\right)\right|-2\left|E\left(L_{i}\right)\right|=2\left|E\left(G_{i}\right)\right|$ for each $i$, and hence $\beta(\mathcal{L})$ divides $\beta(\mathcal{G})$. This conffirms (3.2).

\section{References}

1. Yeow Meng Chee, Charles J. Colbourn, Alar C. H. Ling, and Richard M. Wilson, Covering and Packing for Pairs, Inamuscript.

2. Chaxles J. Colboum and Vojtech Rödl Percentages in pairuise balanced designs, Discrete Matherratics 77 (1989), 57-683. 
3. Eisther Lamken and Fichard M. Wilson, Decompostions of edge-colored complete grophs, J. Combin. Theory Ser. A $89(2000), 449-200$.

4. Richsird M. Wilson, An existence theory for patrwise balanced designs, WT. Proof of the existence conjectures, J. Combinatorial Theory Ser. A 18 (1975), 71 . 79 .

DEPARTMEN' OF MATEEMATIG, CALFORNA INSTTUTE OF TEChNOLOGY, PASADENA, CALI FORNIA, 91125

Camrent address: Departmert of Wathematics, 253-37, Calfornia Institute of Technology, Pasadena, California, 91125

Email address: rmwealtech.adu 